\title{
Properties of the Near-Earth object population: the ACM 2005 view
}

\author{
Richard P. Binzel ${ }^{1}$ and Dimitrij F. Lupishko ${ }^{2}$ \\ ${ }^{1}$ Department of Earth, Atmospheric, and Planetary Sciences, Massachusetts Institute \\ of Technology, Cambridge, MA 02139, USA \\ email: rpb@mit.edu \\ ${ }^{2}$ Institute of Astronomy of Karazin Kharkiv National University, Ukraine \\ email: lupishko@astron.kharkov.ua
}

\begin{abstract}
Within the near-Earth object population, one finds asteroids, comets, and meteorites thereby placing the NEO population at the center of the ACM conference. The longstanding gulf between the spectral properties of S-type asteroids and ordinary chondrite meteorites appears to be bridged, where the observational data are consistent with a space weathering type process. As much as $30 \%$ of the entire NEO population may reside in orbits having a Jovian Tisserand parameter $<3$, and among these roughly half are observed to have comet-like physical properties in terms of their albedos and spectra (taxonomy). Thus $15 \pm 5 \%$ of the entire NEO population may be comprised by extinct or dormant comets.
\end{abstract}

Keywords. steroid, comet, meteorite, spectra, albedo

\section{Introduction}

Near-Earth objects (NEOs) are defined as asteroids and comets having orbits with perihelion distances of $1.3 \mathrm{AU}$ or less. More and more it is becoming apparent that an understanding of this population requires increasing focus on the interrelationships between the broad classes of objects found in near-Earth space. What are the relationships between asteroids and meteorites? What are the relationships between asteroids and comets? In essence, these are questions at the very heart of the Asteroids Comets Meteors conference themes, placing the study of NEOs at ACMs center stage.

This review, intended to give only a brief overview, will focus primarily on the progress made since the Berlin ACM meeting in 2002. In particular, this review addresses the two fundamental questions: How are meteorites related to asteroids? How are asteroids related to comets? This review will focus on spectroscopic and albedo properties as rotational properties of asteroids, including NEOs, are covered elsewhere.

\section{Progress in Measurements}

The population of known NEOs is constantly increasing owing to the continuing rapid pace of discovery. Yet observers performing physical studies of NEOs have done an excellent job of keeping pace with discoveries, as shown in Figure 1. A great increase in the number of spectral measurements of NEOs from European sponsored observatories at La Palma and the European Southern Observatory (ESO) has been responsible for much of the growth (e.g. Lazzarin et al. 2005; Lazzaro et al. 2004; Dandy et al. 2003; Vernazza et al. 2004). Albedo measurements have similarly kept pace, where measurements from Mauna Kea (e.g. Delbó et al. 2003; Wolters et al. 2005; Fernandez et al. 2005) have been the key to this growth. It will become increasingly challenging for physical 


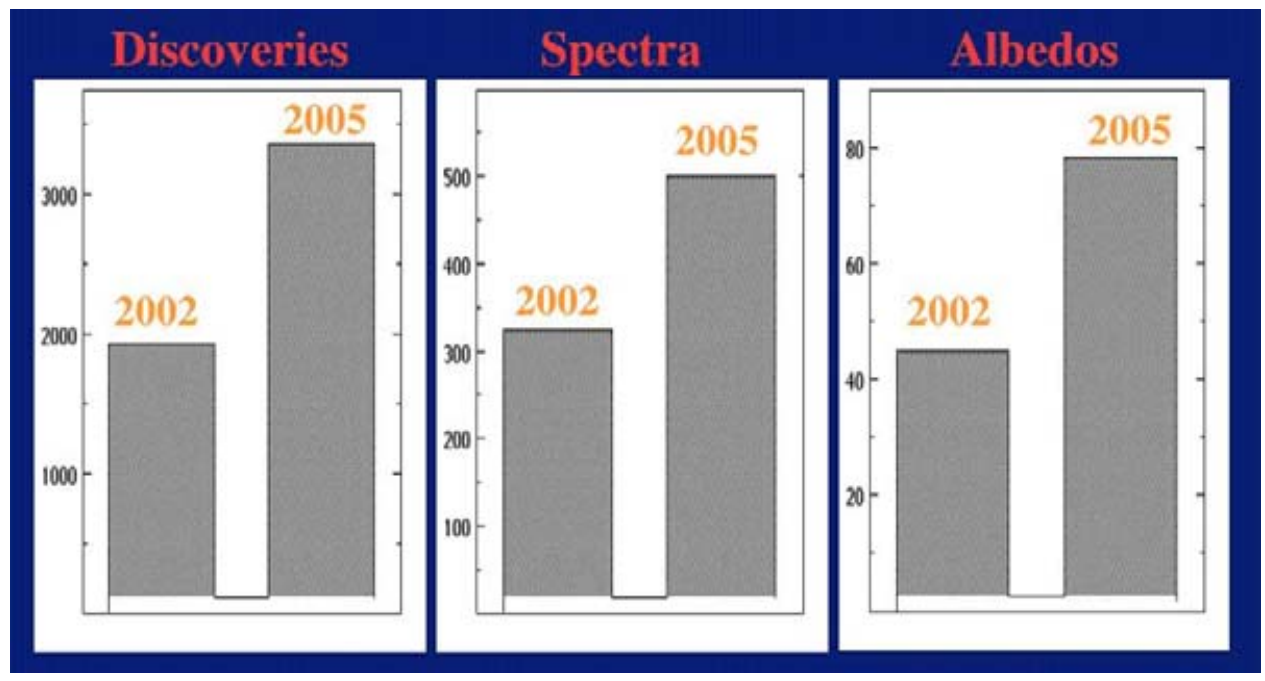

Figure 1. Histograms showing the relative growth in the number of NEO discoveries from 2002 (ACM in Berlin) to 2005 (ACM in Buzios) and the relative growth in measurements of their spectral and albedo properties. As noted in the text, the greatest growth in physical measurements has come from European sponsored observatories.

studies of NEOs to keep pace with discoveries as search surveys push to fainter limiting magnitudes, requiring physical observers to compete for access on larger telescopes. All available data on NEOs confirm an early finding by McFadden et al. (1984) that NEOs show the same diversity, if not more, of the main-belt asteroid population (Binzel et al. 2002). Such diversity means that unusual taxonomic types present in the main-belt, such as olivine-pyroxene mixture R-types, are also found in the near-Earth population (Marchi et al. 2005; Binzel et al. 2004a). Additionally, previously rare objects such as V-types, possibly related to asteroid 4 Vesta (which is thought to be differentiated) and eucrite meteorites, represent $\sim 7$ percent of all classified NEOs (Marchi et al. 2005). Particularly interesting are the physical properties of the smallest objects, observable because of their proximity to Earth. At the small sizes, the likelihood increases that an object samples a distinct geologic unit of a parent body or that the object may be regolith free bare rock. Approaching the Earth gives a unique opportunity to observe them at a much wider range of geometries and illumination than for MBAs, thereby such observations of NEOs also help us to study the MBAs of corresponding types. For example, polarimetric observations of the E-type Aten-object (33342) 1998 WT24 with albedo $\mathrm{p}_{v}=0.43$ have resulted in a complete phase angle dependence of polarization for high-albedo E-type asteroids (Kiselev et al. 2002). That dependence showed an extremely small maximum positive polarization (1.7\%), possibly indicative of an absorbing or somewhat peculiar (glassy?) surface. The second peculiarity of polarization-phase dependence of E-type asteroids is a so-called polarization opposition effect, recently revealed for the bright Jovian satellites and E-asteroids (Rosenbush 2005). NEO physical studies, which up till now have largely operated in survey mode, will likely become increasingly focused on understanding the detailed properties of individual objects.

\section{Comparisons to Meteorites}

By definition, a body entering the atmosphere and delivering meteorites is a nearEarth object prior to its arrival. Thus, the study of NEOs provides an opportunity to 
examine the population in space that includes precursors to meteorites. By virtue of their proximity, NEOs are the smallest individually observable objects in space. As physical studies have progressed to smaller and smaller sizes, better and better understanding of correlations to meteorite classes have been achieved - even for rare meteorite types. In addition to the eucrites mentioned above, rare types apparently related to aubrite meteorites (enstatite achondrites, consisting of enstatite with very low content of highly reduced Fe) have been increasingly studied. The E-type NEO 3103 Eger is the earliest recognized candidate related to the aubrites (Gaffey et al. 1992), with most recent studies focusing on its detailed mineralogy (Burbine et al. 2002; Gaffey \& Kelley 2004). Additional aubrite candidates include 4660 Nereus (Binzel et al. 2004b), a particularly exciting prospect owing to its relatively accessible orbit for spacecraft exploration.

The historically most troublesome asteroid-meteorite connection has been for the most common meteorites, the ordinary chondrites (see the review by Clark et al. 2002 and references therein). Most logically, the most common meteorites should be related to the most commonly observed asteroids (and NEOs), those falling into the S-class. However, a distinct mismatch in their spectral characteristics, most notably their spectral slopes, has prevented any clear correlation. Pushing to smaller sized objects among NEOs proved fruitful in resolving this problem as the distinct difference between S-type spectra and ordinary chondrite spectra was found to be "filled" by a continuous range of NEO spectral properties when examined over visual wavelengths (Binzel et al. 1996). Extending these observations over near-infrared wavelengths continues to confirm this continuum, as shown by Binzel et al. (2001; see Figure 6) and further updated in Figure 2, presented here. This continuum is interpreted as being in favor of some type of space weathering process (Clark et al. 2002) where ordinary chondrite-like asteroid surfaces are altered over time to look more and more like S-type asteroids. By far the most convincing evidence for a relationship between S-asteroids and ordinary chondrite meteorites is the finding by the NEAR mission of ordinary chondrite-like elemental abundances for the S-type asteroid 433 Eros (Trombka et al. 2000). A physical explanation for space weathering, proposed by Hapke et al. (1975) and identified in the laboratory by Pieters et al. (2000) involves the coating of silicate grains by nanometer scale particles of Fe, created in the vaporization of small Fe particles by micrometeorite impacts. Ongoing laboratory work (Moroz et al. 1996; Sasaki et al. 2001; Kurahashi et al. 2002; Strazzulla et al. 2005) shows good support for this process and may explain why ordinary chondrites may be more susceptible to space weathering than carbonaceous chondrites or eucrites. The key ingredient appears to be the availability of metallic Fe in the surface which is present for ordinary chondrites, but not for achondrite meteorites such as eucrites nor for primitive meteorites such as carbonaceous chondrites.

Further support for an age dependence, where young "fresh" surfaces look more like ordinary chondrite meteorites comes from finding ordinary chondrite-like NEOs (denoted as objects having Q-type spectra) preferentially among the smaller-sized NEOs (Angeli \& Lazzaro 2002; Dandy et al. 2003; Lazzarin et al. 2005). Binzel et al. (2004a) find an explicit diameter dependence, illustrated in Figure 3, that appears to show NEOs larger than $5 \mathrm{~km}$ predominantly being "S-type asteroids" with spectral properties very similar to those observed for S-types among main-belt asteroids. Such a trend is consistent with space weathering models because on average, small objects have shorter collisional lifetimes (before they are destroyed) compared with larger objects. Thus younger "fresher" surfaces are more commonly found among the smallest objects. (In fact, the smallest objects should have the greatest range of variation as this group is most likely to include both fresh surfaces as well as some objects that have been fortunate to survive somewhat longer than average.) While a diameter dependent spectral trend is consistent with a 


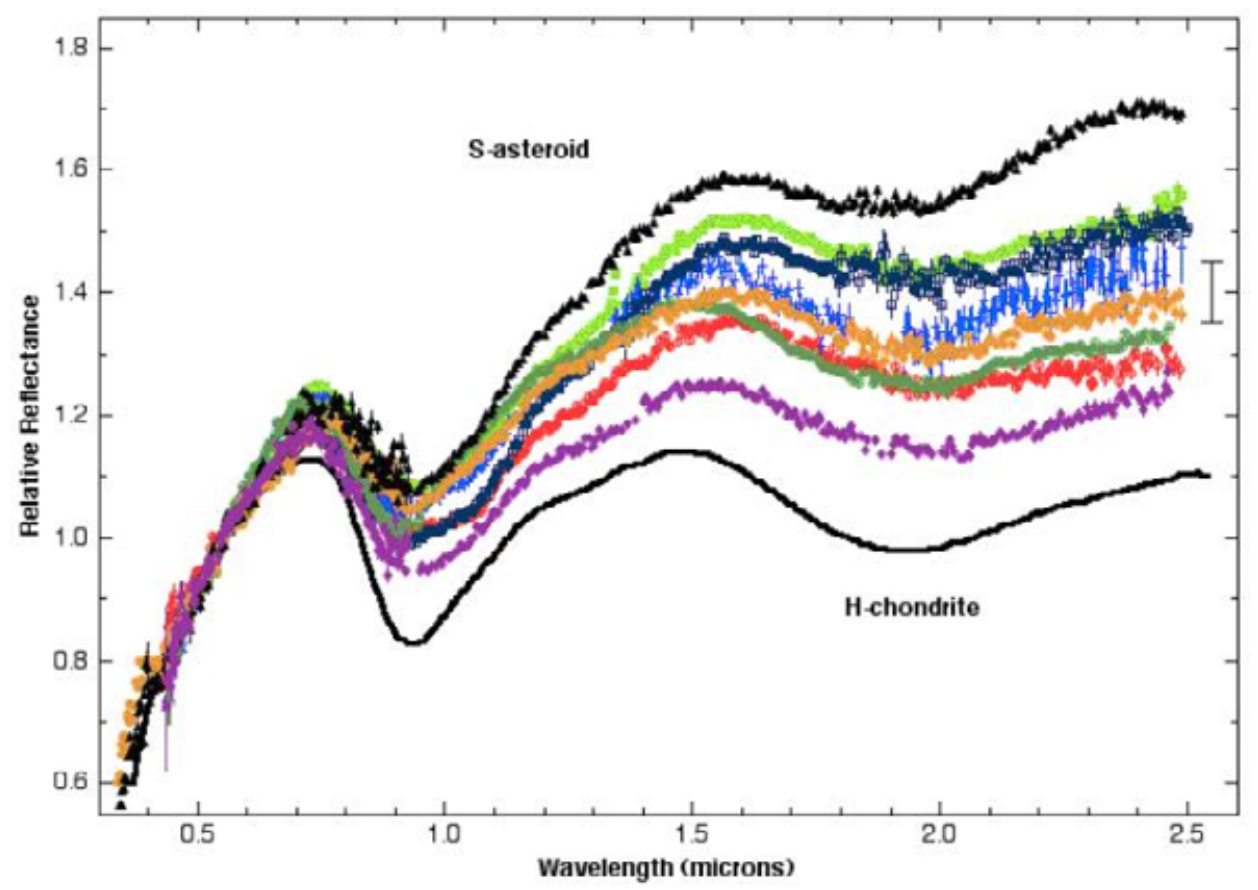

Figure 2. Historically, S-type asteroids have been shown to be distinctly separate from those of ordinary chondrite (OC) meteorites, with the exception of Q-type asteroids matching the OC meteorites. Binzel et al. $(1996,2001)$ found a full continuum of spectral properties between S-type asteroids and ordinary chondrite meteorites. The data displayed here (extending out to 2.5 microns) show a further update on this continuum. These data, obtained with the SpeX instrument on the NASA IRTF, are part of a MIT-Hawaii-IRTF joint program for NEO reconnaissance for which NEO spectral data are made freely available to the community in near-real time after observation. Full information is available via http://smass.mit.edu

surface age space weathering process, outstanding questions remain: How much of this trend is actually the result of increasing gravity improving the retention of a regolith? Under the Hapke et al. (1975) - Pieters et al. (2000) description of space weathering, the presence of a regolith is a requirement for the process to be effective. The relative roles of the weathering process, gravity, and regolith retention remains an unsolved problem that may be particularly inviting to new researchers.

Overall, it is also possible to suggest that the taxonomic classification and mineralogical interpretation of NEO spectra show evidence of genetic relationship between NEOs and main-belt asteroids. Interestingly, most of the NEOs characterized to date represent differentiated assemblages. Among the NEOs there are bodies with monomineral silicate composition and purely metallic ones. For example, small asteroid 1915 Quetzalcoatl appears to have little or no olivine, and diogenitic meteorites (Mg-pyroxenes) are possible analogs of it. 3199 Nefertiti has the same content of pyroxene and its composition corresponds to that of stony-iron meteorites pallasites (metal + olivine). There are three M-objects, one of which, (6178) 1986 DA has a high radar albedo (0.58) clearly indicating the real metallic composition of this asteroid. 3103 Eger with a very high albedo (0.53) may correspond to assemblages of iron-free silicate minerals, such as enstatite. More than 20 NEOs classified as V-class, have spectra matching the main-belt asteroid 4 Vesta, which is known to be a differentiated body covered by basaltic (pyroxene-rich) 


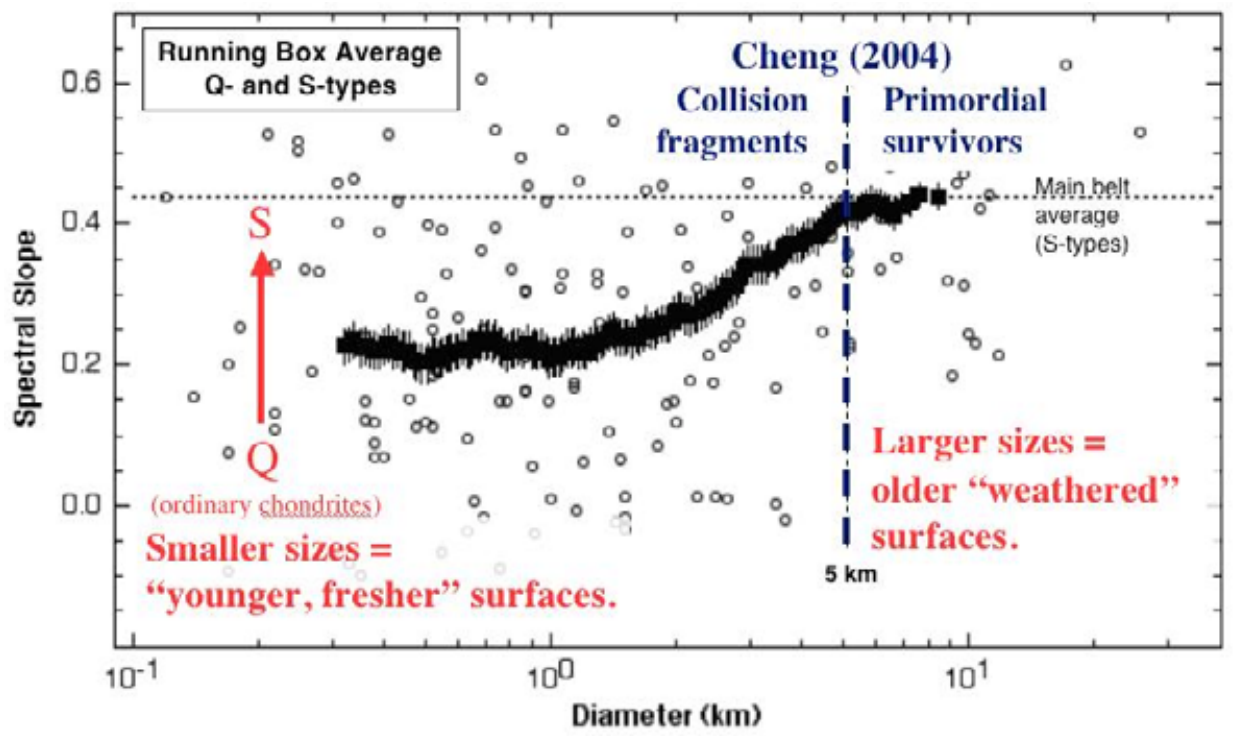

Figure 3. Individual measurements (circles) of spectral slope for Q- and S-type asteroids are plotted versus estimated diameter. A running box mean (box size $=50$ ) reveals a diameter dependence where objects trend from low spectral slopes (Q-types, resembling ordinary chondrites) to higher spectral slopes displayed by S-types, typical for asteroids in the main-belt (dashed horizontal line). If the trend is due to a space weathering process, this diagram can be interpreted as revealing increasing weathering with increasing size where the age of the object likely increases (more resilient to collisional disruption) with increasing size. The threshold of $5 \mathrm{~km}$ and larger appears to be the size where objects have sufficient age or sufficient gravity for regolith retention so that space weathering processes are complete. Independently, Cheng (2004) finds that this same threshold $(5 \mathrm{~km})$ may mark the boundary between primordial survivors and multi-generation fragments among the asteroids.

material. About $30 \%$ of NEAs belong to Q-types which are the ordinary chondrite-like objects. Thus the variety of taxonomic classes among NEOs reflects the diversity of their surface mineralogy and an overall analogy with the main belt. Taking into account their small sizes, one might infer that they are the fragments of much larger differentiated bodies which were later injected into the present orbits.

\section{Comet Fraction Within the NEO Population}

"What is the source of the NEO population?" is one of the most fundamental questions for the field. The question arises because NEOs have relatively short dynamical lifetimes $\left(10^{6}-10^{7}\right.$ years $)$, meaning that the population we see today must have some source of resupply. (Any primordial objects residing in near-Earth space at the beginning of the solar system have long since been removed). Many observational factors indicate the mainbelt as their predominant source: their general matching of taxonomic and mineralogic distributions - especially for differentiated assemblages, their approximate same shapes and rotation, and their overall similar optical properties and surface structure. This conclusion does not contradict the recent results of dynamic considerations, according to which the main asteroid belt can supply a few hundred km-sized NEAs per $1 \mathrm{Myr}$, well enough to sustain the current population of asteroid-like NEOs (Menichella et al. 1996). 
While transport of main-belt asteroid fragments via the resonances likely dominates the NEO supply source, some fraction likely enters as nuclei of short-period comets. These objects having a cometary origin, if inactive at the time of discovery, are catalogued as "asteroidal" objects. We seek to answer the question: what fraction of the NEO population, having an "asteroidal appearance" are actually extinct or dormant comets? We apply both dynamical and observational criteria to address this question.

The candidates for cometary origin, as a rule, should satisfy the following conditions: (a) they should be low-albedo objects of D-, P- and C-types (Hartmann et al. 1987); (b)their rotational rates should be lower than the mean rates of NEOs (Degewij \& Tedesco 1982; Hartmann \& Tholen 1990; Weissman et al. 2002); (c) they should also be evolved on unstable orbits, and should be associated with meteor streams (Weissman et al. 1989). The data available (NEO taxonomy, shapes and rotation parameters, associations with meteors streams) allowed Lupishko \& Lupishko (2001) to conclude that no more than $10 \%$ of NEAs have cometary origin. A convergence of similar results is coming from many researchers: Fernandez et al. (2001) estimate “... at least $9 \%$ of NEOs are cometary nuclei"; Whiteley (2001) estimates "... on the order of $5 \%$ of cometary origin"; Bottke et al. (2004) estimate $\sim 6 \%$ of NEOs comes from the Jupiter-family comet region $(2<T<3)$.

For this review, we use the Jovian Tisserand parameter $(T)$ as our dynamical criterion for identifying potential comet candidates among asteroidal NEOs. We consider dynamical comet candidates to be objects having $T<3$, because this indicates that they reside in orbits that are strongly influenced by Jupiter, as exemplified by Jupiter family comets. Consistent with this dynamical criterion is the classic discovery by Fernandez et al. $(2001,2005)$ that objects with $T<3$ have, on average, substantially lower albedos than objects with $T>3$. The predominance of low albedos among $T<3$ objects has extremely important implications for statistically evaluating the fraction of comet candidates within the total NEO population: Our discovery statistics are severely biased against discovering these types of objects. The bias arises because a low albedo (and correspondingly fainter apparent magnitude relative to a high albedo object of the same size) decreases the likelihood of $T<3$ objects being discovered. What's more, the larger orbital eccentricities (a factor in reaching $T<3$ values) also biases against $T<3$ NEO discoveries since such objects spend greater fractions of their orbital period at aphelion (where they are more difficult to discover) than at perihelion. Thus a bias corrected estimate for the total population of $T<3$ objects is required before any overall estimate of the comet fraction can be made. Fortunately, such bias corrected estimates are available through the Ph.D. thesis work of J. Scott Stuart (2003; see also Stuart \& Binzel 2004). The debias work of Stuart (2003), based on the extensive search statistics of the LINEAR survey, focuses on determining the size distribution of the total NEO population. One outcome of Stuarts work, key to our analysis, is that the bias correction shows that $30 \%$ of all NEOs reside in orbits having $T<3$.

If $30 \%$ of the total NEO population satisfies the $T<3$ criterion as extinct comet candidates, what fraction of these have physical properties that make them "look" like comets? Based on the fact that measured albedos for comet nuclei also have low values, Fernandez et al. (2005) set $<0.075$ as a reasonable albedo criterion for identifying objects that look like "comets". Binzel et al. (2004a) recognized that relatively few albedo data exist compared with spectral data and used taxonomic class (specifically C-, D-, and Ptypes) as a proxy for identifying low albedo NEOs within the $T<3$ population, finding $50 \pm 10 \%$ of observed $T<3$ NEOs have "comet-like" physical properties. Fernandez et al. (2005) applied the $<0.075$ albedo criterion and found $53 \pm 9 \%$ as the observed "cometlike" fraction among $T<3$ NEOs. These independent physical estimates of comet-like properties for $50 \%$ of observed $T<3$ objects, convolved with the Stuart debiased result 
that $30 \%$ of all NEOs reside in $T<3$ orbits, yields:

$$
0.50 \times 0.30=0.15
$$

implying $15 \pm 5 \%$ as the total fraction of the NEO population that has both dynamical and physical characteristics consistent with their being dormant or extinct comets.

It is worth emphasizing that this $15 \%$ estimate for the extinct or dormant comet fraction is a debiased or diameter-limited estimate. (Many previous, slightly lower estimates are magnitude limited, with no debias criteria applied. Estimates not compensated for the bias against the discovery and measurement of low albedo objects will tend to lead to underestimates.) It is also important to emphasize that our $15 \%$ is just a broad characterization of the population as a whole. Certainly there are other criteria (e.g. meteor stream correlations, etc.; see Weissman et al. 1989) that allow objects with $T>3$ to be comet candidates. Objects in the inner solar system, gravitationally interacting with the terrestrial planets, can have their coupling with Jupiter altered so that a $T<3$ orbit evolves to $T>3$. Similarly, asteroids entering near-Earth space with $T>3$ can be perturbed into $T<3$ orbits. Thus the $15 \pm 5 \%$ estimate for comet candidates within all of the NEO population does not tell the complete story of asteroid-comet connections among NEOs, but does likely provide fertile ground for detailed study of possible low level cometary activity or perhaps attractive destinations for mission opportunities.

\section{Concluding Remarks}

Near-Earth objects have become increasingly central to the study of solar system small bodies by the ACM community. Progress in their measurement and understanding shows they are a diverse population containing both asteroidal bodies from the main-belt as well as a possibly substantial fraction of extinct comet nuclei. Our improved understanding of their meteorite connections, when combined with our understanding of their shapes and rotations (described in this volume), shows their internal structures may be diverse as well. The smallest and fastest rotating bodies may be intact with substantial internal strengths, while the larger and more slowly rotating bodies may be strengthless rubble piles held together only by their mutual gravity. Understanding the internal properties of small bodies, of which NEOs are the most easily accessible to spacecraft missions, is becoming an increasing focus for scientific investigation. Not only does this represent a new area for scientific curiosity, but it is an area of practical responsibility should we some day discover an NEO with a certainty of collision in the coming decades. Thus for both science reasons and practical reasons, the study of NEOs will remain highly important to the ACM community in the future.

\section{Acknowledgements}

The research of the first author is supported by grants from NASA and the National Science Foundation. D. Lupishko sincerely thanks the LOC of ACM 2005 (IAU Symposium 229) for the grant allowing him to participate in the meeting.

\section{References}

Angeli, C.A. \& Lazzaro, D. 2002, Astron. Astrophys. 391, 757

Binzel, R.P., Bus, S.J., Burbine, T.H., \& Sunshine, J.M. 1996, Science 273, 946

Binzel, R.P., Harris A.W., Bus S.J., \& Burbine, T.H. 2001, Icarus 151, 139

Binzel, R.P., Lupishko D.F., Di Martino M., Whiteley R.J., \& Hahn G.J. 2002, in: W. Bottke, A. Cellino, P. Paolicchi, and R. Binzel, (eds.), Asteroids III (Tucson: Univ. Arizona Press), p. 255 
Binzel, R.P., Rivkin, A.S., Scott, J.S., Harris, A.W., Bus, S.J., \& Burbine, T.H. 2004a, Icarus 170,259

Binzel, R.P., Birlan, M., Bus, S.J., Harris, A.W., Rivkin, A.S., \& Fornasier, S. 2004b, Plan. Space Sci. 52, 291

Bottke, W.F. Jr., Morbidelli, A., \& Jedicke R. 2004, in: M.J.S Belton, T.H. Morgan, N.H. Samarasinha, and D.K. Yeomans (eds.), Mitigation of Hazardous Comets and Asteroids (Cambridge: Univ. Press), p. 1

Burbine, T.H., McCoy, T.J., Nittler, L.R., Benedix, G.K., Cloutis, E.A., \& Dickinson, T.L. 2002, Met. Plan. Sci. 37, 1233

Cheng, A.F. 2004, Icarus 169, 357

Clark, B.E., Hapke, B., Pieters, C., \& Britt, D. 2002, in: W. Bottke, A. Cellino, P. Paolicchi, and R. Binzel (eds.), Asteroids III (Tucson: Univ. Arizona Press), p. 585

Dandy, C.L., Fitzsimmons, A., \& Collander-Brown, S.J. 2003, Icarus 163, 363

Degewij, J. \& Tedesco, E.F. 1982, in: L. Wilkening (ed.), Comets (Tucson: Univ. Arizona Press), p. 665

Delbó, M., Harris, A.W., Binzel, R.P., Pravec, P., \& Davies, J.K. 2003, Icarus 166, 116

Fernandez, Y.R., Jewitt, D.C., \& Sheppard, S.S. 2001, Astrophys. J. 553, L197.

Fernandez, Y.R., Jewitt, D.C., \& Sheppard, S.S. 2005, Astron. J. 130, 308

Gaffey, M.J., Reed, K.L., \& Kelley, M.S. 1992, Icarus 100, 95

Gaffey, M.J. \& Kelley, M.S. 2004, Lunar Plan. Sci. Conf. Abstract 1812

Hartmann, W.K., Tholen, D.J., \& Cruikshank, D.P. 1987, Icarus 69, 33

Hartmann, W.K. \& Tholen, D.J. 1990, Icarus 86, 448

Hapke B., Cassidy W., \& Wells E. 1975, Moon 13, 339

Kiselev, N.N., Rosenbush, V.K., Jockers, K., Velichko, F.P., Shakhovskoj, N.M., Efimov, Yu.S., Lupishko, D.F., \& Rumyantsev, V.V. 2002, Proc. of the Conf. Asteroids, Comets, Meteors 2002 (Berlin:Techn. Univers.), p. 887

Kurahashi, E., Yamanaka, C., Nakamura, K., \& Sasaki, S. 2002, Earth Plan. Space 54, e5

Lazzarin, M., Marchi, S. Magrin, S., \& Licandro, J. 2005, MNRAS 359, 1575

Lazzaro, D., Angeli, C.A., Carvano, J.M., Mothe-Diniz, T., Duffard, R., \& Florczak, M. 2004, Icarus 172,179

Lupishko, D.F. \& Lupishko, T.A. 2001, Solar System Research 35, 227

Marchi, S., Lazzarin, M., Paolicchi, P., \& Magrin, S. 2005, Icarus 175, 170

McFadden, L.A., Gaffey, M.J., \& McCord, T.B. 1984, Icarus 59, 25

Menichella, M., Paolicchi, P., \& Farinella, P. 1996, Earth, Moon, and Planets 72, 133

Moroz, L.V., Fisenko, A.V., Semjonova, L.F., Pieters, C.M., \& Korotaeva, N. 1996, Icarus 122, 366

Pieters, C.A., Taylor, L.A., Noble, S.K., Keller, L.P., Hapke B., Morris, R.V., Allen, C.C., McKay, D.S., \& Wentworth, S. 2000, Meteoritics 83 Planet. Sci. 35, 1101

Rosenbush, V.K., Kiselev, N.N., Shevchenko, V.G., Jockers, K., Shakhovskoy, N.M., \& Efimov, Y.S. 2005, Icarus 178, 222

Sasaki, S., Nakamura, K., Hamabe, Y., Kurahashi, E., \& Hiroi, T. 2001, Nature 410, 555

Strazzulla, G., Dotto, E., Binzel, R., Brunetto, R., Barucci, M.A., Blanco, A., \& Orofino, V. 2005, Icarus 174, 31

Stuart, J.S. 2003, PhD thesis, Massachusetts Institute of Technology, Cambridge.

Stuart, J.S. \& Binzel, R.P. 2004, Icarus 170, 295

Trombka J., Squyres, S., Bruckner, J., Boynton, W., Reedy, R., McCoy, T., Gorenstein, P., Evans, L., Arnold, J., Starr, R., Nittler, L., Murphy, M., Mikheeva, I., McNutt, R., McClanahan, T., McCartney, E., Goldsten, J., Gold, R., Floyd, S., Clark, P., Burbine T., Bhangoo, J., Bailey, S., \& Pataev, M. 2000, Science 289, 2101

Vernazza, P., Fulchignoni, M., \& Birlan, M. 2004, 35th COSPAR Sci. Assembly 2456.

Whiteley, R.J. 2001, PhD thesis, University of Hawaii.

Weissman, P.R., AHearn, M.F., MacFadden, L.A., \& Rickman, H. 1989, in: R.P. Binzel, T. Gehrels, and M.S. Matthews (eds.), Asteroids II) (Tucson: Univ. Arizona Press), p. 880

Weissman, P.R., Bottke, W.F., \& Levison, H.F. 2002, in: W. Bottke, A. Cellino, P. Paolicchi, and R. Binzel (eds.) Asteroids III, (Tucson: Univ. Arizona Press), p. 669

Wolters, S.D., Green, S.F., McBride, N., \& Davies, J.K. 2005, Icarus 175, 92 